\title{
Design of Wireless Intelligent Greenhouse Control System Based on Embedded Technology
}

\author{
Jianling Qi, Hang su, Xiaodong Duan, Shuya Zhao \\ North China institute of aerospace engineering, Langfang, China \\ E-mail: qjl67@163.com
}

Keywords: greenhouse control, STM32 controller, temperature collection, remote control

Abstract. This paper introduces a kind of intelligent greenhouse measurement and control system based on embedded technology and wireless communication technology. The hardware and software control flow of the system are described. In this paper, the method of measuring and controlling the conventional environmental parameters in greenhouse is presented, and the design method of the upper computer and lower computer is introduced.

\section{Introduction}

In recent years, with the rapid development of industrial automation technology and network technology, agricultural technology has entered a new era of rapid development, intelligent agriculture has become the research point of the national science and technology. The use of advanced facilities and technology of greenhouse production, become an important way of intelligent agricultural practices. Due to the limitations of various conditions, it is difficult to carry out large-scale greenhouse cultivation in China. Therefore, it is of great significance to study the intelligent environment monitoring system for medium and small greenhouse planting.

Based on the summary of various kinds of greenhouse intelligent control system, a low cost medium and small intelligent greenhouse monitoring and controlling system is developed in this paper.

\section{The composition of Intelligent Greenhouse Monitoring and controlling system}

Intelligent greenhouse measurement and control system is shown in Fig. 1, mainly by the following parts:(1) Upper computer: Including STM32 microcontroller, keyboard, display, sensor, actuator and wireless communication module, etc. (2) Lower computer: From industrial control computer and wireless communication module, mouse, keyboard and so on. (3) Remote client: Consists of computer, tablet computer and mobile phone connected to internet.

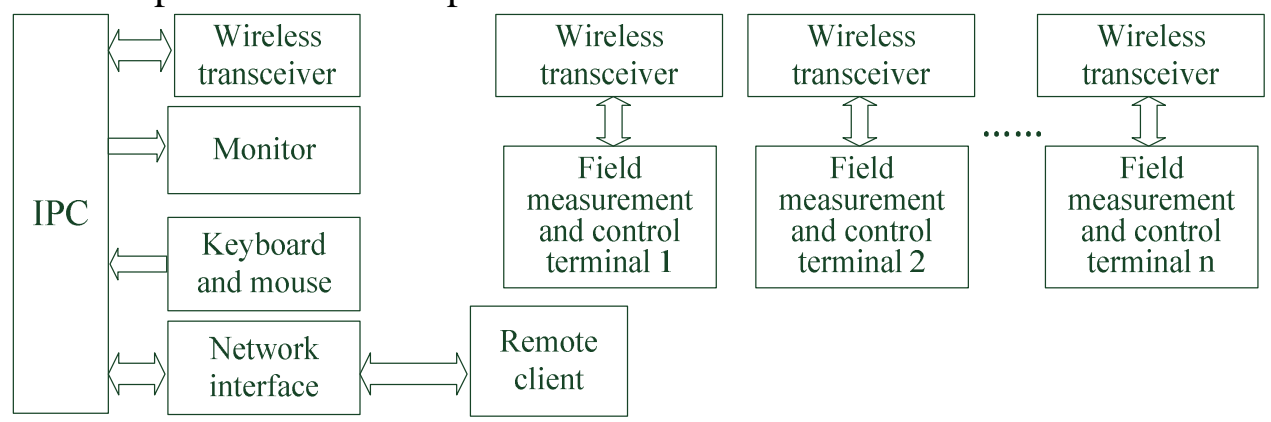

Fig. 1: structure diagram of greenhouse control system

The part of lower computer. The part is deployed in each greenhouse, and the greenhouse environment variable is collected and controlled in real time, each of the parameters and control devices in the greenhouse can be monitored by the display and keyboard, and the system can be automatically controlled by the setting of various parameters. The controller will be collected from the environmental parameters and the state of the controlled device is displayed on the TFT LCD 
screen. At the same time, it can be transmitted to the host computer through the wireless communication module, so as to realize the dual control of the local and remote.

The part of upper computer. The upper computer is a computer equipped with configuration software. The data exchange with the lower computer is carried out by the wireless transceiver; it can set the environmental parameters of the greenhouse, the running status of field equipment and environmental data are monitored and stored. Users can extract and print historical data according to the need.

Remote client. Everything can be connected to the Internet's interactive devices can become the system remote client. Through the Internet access to the IP address of the upper computer, the configuration screen of the upper computer configuration software can be obtained. All information related to the greenhouse control can be obtained by using the configuration screen; User with permission can also configure the field environment parameters through the buttons on the interface or control of field equipment.

System hardware design. System hardware overall design scheme is shown in Fig. 2. Controller based on Cortex kernel STM32F103ZET6, it has a 72M frequency and rich on-chip peripherals. Both to meet the requirements of the system for high-speed computing, but also save a lot of unnecessary hardware overhead; at the same time, the variety of communication interfaces on the chip enhance the scalability and the real-time performance of data acquisition.

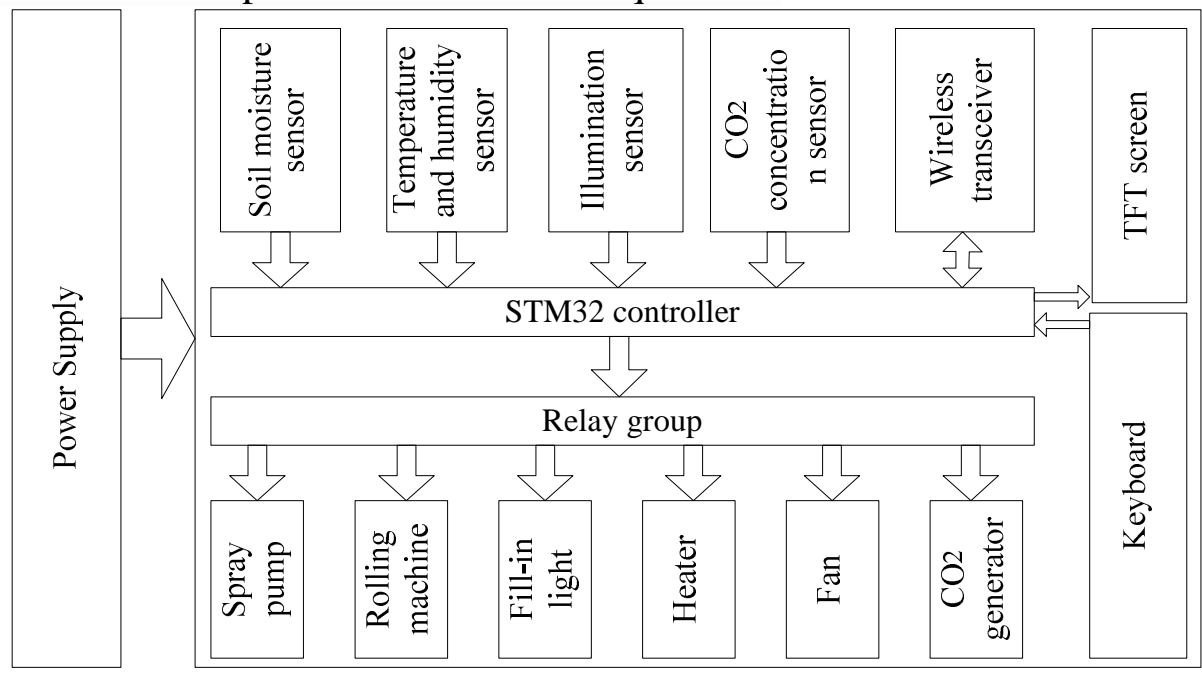

Fig. 2: System overall design schemes

Power module. Power module with multiple output supply for the controller (3.3V), the relay group $(12 \mathrm{~V})$, and the sensor $(5 \mathrm{~V})$, respectively, it can meet the requirement of voltage and power, but also realize the isolation between different parts.

Relay module. Controller for spray pump, rolling machine, heater, fan and $\mathrm{CO}_{2}$ generator control is achieved through the relay. This control mode can realize the control of the power device with the small power device; it also has the characteristics of high reliability and simple control. Relay module circuit connection diagram is shown in Fig. 3. The U1 is a photoelectric coupler, which can realize the electrical isolation of the relay, and reduce the disturbance of the controller when the device is on and off. 


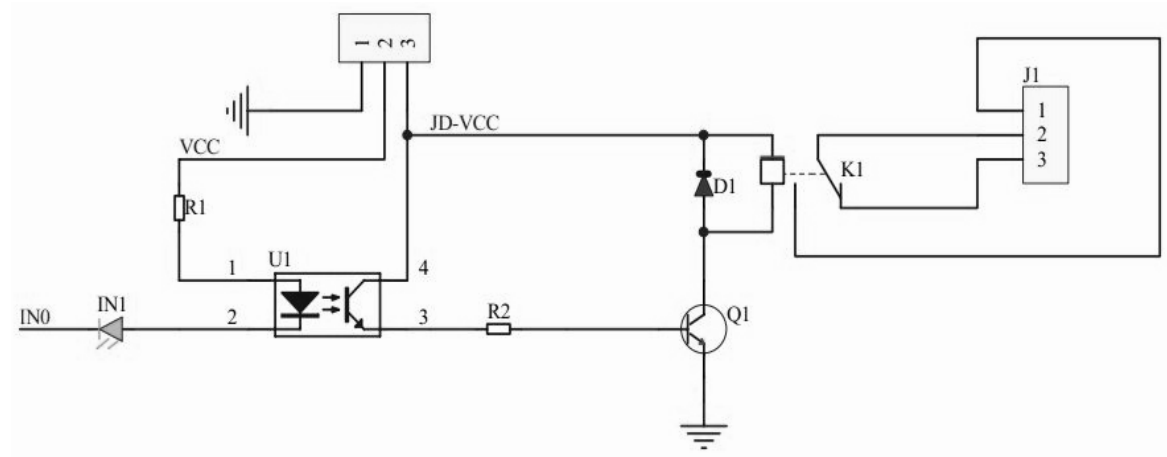

Fig. 3: Schematic diagram of relay module

PWM lighting circuit. The system adopts the PWM mode control lights. The controller generates the PWM signal to control FET on and off, so as to realize the continuous adjustment of the brightness of the lights. As shown in Fig. 4.

Soil moisture and light intensity measurement circuit. Soil moisture was measured indirectly by measuring the soil resistance. The soil moisture sensor converts the resistance of the soil into a voltage signal. The soil moisture value can be obtained by the conversion of the AD converter and the correction process.

The light intensity of the soil was measured by photosensitive resistance, measurement of light intensity by a similar method with soil moisture.

Air temperature and humidity measuring circuit. Air temperature and humidity measurement are completed using temperature and humidity integrated digital sensor DHT11. The sensor directly outputs temperature and humidity after calibration, through the data bus in the form of digital quantity. Sensor and controller of the connection is simple, and have strong anti-interference performance. As shown in Fig. 5.

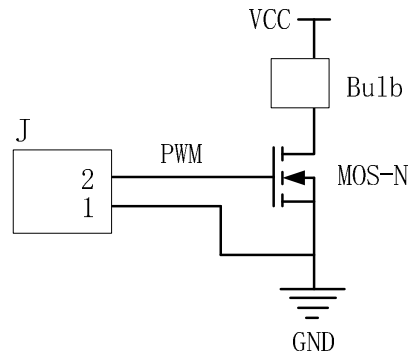

Fig. 4: PWM lighting circuit

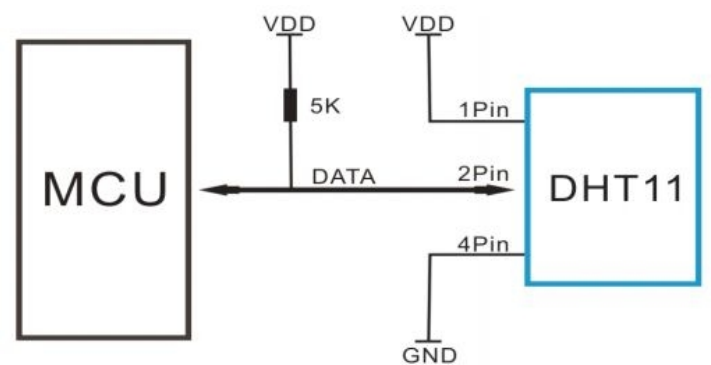

Fig. 5: The connection mode of DHT11 and controller

$\mathrm{CO}_{2}$ concentration measurement circuit. The measurement of $\mathrm{CO}_{2}$ concentration is achieved by the sensor module MG811. It can detect the gas concentration range of 0 10000ppm, the higher the $\mathrm{CO}_{2}$ concentration, the lower the output voltage. It can achieve the dual signal output of analog signal and TTL level. 30 50mV analog voltage output signal is used in this paper. The concentration of $\mathrm{CO}_{2}$ in the greenhouse was acquired by amplifying circuit and A/D conversion circuit.

Wireless communication module. In order to achieve the point to point communication to a single greenhouse, and the network communication to a number of greenhouse monitoring, taking into account the communication distance, the system uses Zigbee wireless communication module SZ05-ZBEE. The module has integrated some of the complex network and communication protocols. Controller only through the serial port and the module can be easily connected to achieve data communication. Controller only through the serial port to connect the module can easily achieve data communication.

\section{Software design}

Program structure. According to the function of the system, the program can be divided into the whole control part and the individual function blocks. The main program completes the control 
process by calling and coordination of various functional blocks. The overall structure of the program is shown in Fig. 6.

Main program flow. The main program flow was shown in Fig. 7. The control of the lower system is realized by calling the function block, in time to receive the control commands sent by the upper computer, the communication part is set up in the interrupt.

\section{Design of configuration software}

The design of configuration software mainly includes the monitoring interface, the command control file, the history record table and the configuration of communication. The monitoring interface completes the display of the time, the setting values of the environmental parameters, environmental parameters, and the state of the equipment, and the parameters are displayed in the form of curves, in order to observe the change of the parameters. The device can also be controlled through the button.



Fig. 6: Program structure diagram

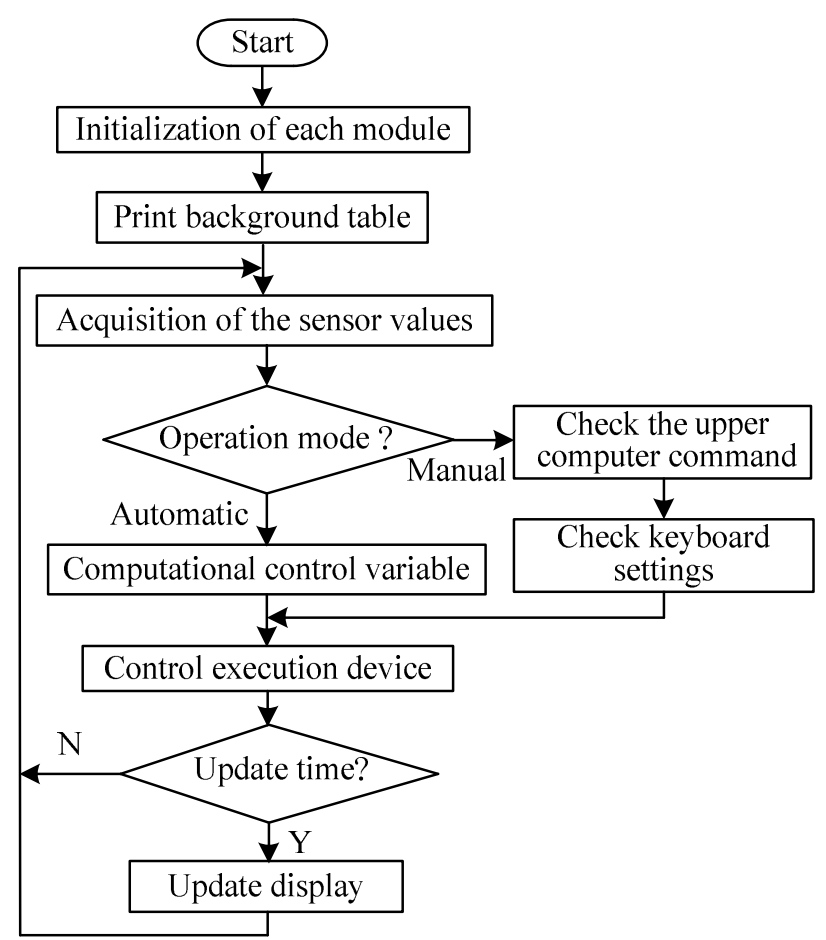

Fig. 7: The main program flow

\section{Conclusions}

This design uses the embedded technology, wireless communication technology and Internet technology. As a new kind of greenhouse control, the method has some advantages and advantages. However, along with the development of the times and people's demand for the improvement of the intelligence, it is bound to expose its limitations and shortcomings. However, based on the fact that the current domestic agricultural automation degree is low, the design has good adaptability to different size and different control requirements, and its low cost; it will occupy a considerable market share in the development of agricultural automation.

\section{References}

[1] Van den Berg G A, Bogers R J. Production planning in closed floriculture systems[C] //Greenhouse Environment Control and Automation1995, ISHS Acta Horticulture 399: 95 100 
[2] Victoria N G, Eveleens B, Van Telgen H J, et al. Critical success factors for robotized rose harvesting[C] //IV International Symposium on Rose Research and Cultivation 2007, ISHS Acta Horticulture 751: 33 42.

[3] Heuvelink E, González-Real M M. Innovation in plant-greenhouse interactions and crop management [C]// International Symposium on High Technology for Greenhouse System Management: Greensys 2007, ISHS Acta Horticulture, 801: 63 74.

[4] Nava S, Tangorra F M, Beretta E, et al. Study and development of an integrated automatic traceability system for the bovine meat chain[C]//7th World Congress on Computers in Agriculture and Natural Resources, Reno, 2009: 367 376.

*This work is supported by scientific research foundation of institute (KYPT-2014-01). 